07,01

\title{
Кинетика развития паттернов макролокализации пластического течения металлов
}

\author{
(С) Л.Б. Зуев, С.А. Баранникова, Б.С. Семухин \\ Институт фризики прочности и материаловедения СО РАН, \\ Томск, Россия \\ E-mail: lbz@ispms.tsc.ru
}

(Поступила в Редакцию 23 января 2018 г.

В окончательной редакции 5 февраля 2018 г.)

\begin{abstract}
Проанализированы особенности макроскопической неоднородности пластической деформации в виде автоволн с пульсирующей амплитудой и приведены данные о локализации источников акустической эмиссии на разных стадиях пластического течения при растяжении ГЦК моно- и поликристаллов. Прослежена взаимосвязь локальных компонент тензора пластической дисторсии в зоне локализации деформации. Рассмотрена роль акустических явлений, сопровождающих локализацию пластической деформации, в развитии процесса пластического формоизменения.
\end{abstract}

Работа выполнена в рамках Программы фундаментальных научных исследований государственных академий наук на 2013-2020 годы.

DOI: 10.21883/FTT.2018.07.46123.020

\section{1. Введение}

В наших работах [1-3] была предложена модель пластического течения, в основе которой лежит эффект самоорганизации дефектной структуры деформируемой среды [4]. Модель основана на общем принципе описания явлений самоорганизации, который состоит в том, что способные к самоорганизации открытые системы самопроизвольно расслаиваются на информационную и динамическую подсистемы. Взаимодействие между подсистемами определяет развитие этого эффекта [5].

При распространении этой идеи на деформируемые среды роль динамической подсистемы, непосредственно ответственной за пластическое формоизменение, была приписана совокупности актов релаксации концентраторов напряжений, таких как заторможенные дислокационные сдвиги с их упругими полями [6]. Информационную подсистему образуют сигналы акустической эмиссии, излучаемые в ходе таких релаксационных актов. В рамках этого подхода процесс пластического течения управляется за счет обмена упругими импульсами между распадающимися (релаксирующими) и возникающими вновь концентраторами напряжений в деформируемой среде. Это отличает развиваемую модель от дислокационных моделей, учитывающих только эволюцию дефектов.

В основе механизма формирования автоволновых мод и соответствующих им паттернов лежит взаимосвязь между элементарными сдвигами и сигналами акустической эмиссии, т.е. между динамической и информационной подсистемами [5]. При пластическом течении импульсы акустической эмиссии перераспределяют упругую энергию между релаксирующими и возникающими при деформации концентраторами. В результате в окрестности последних растут напряжения, повышая вероятность реализации новых термически активированных сдвигов [7].

Для обсуждаемой модели важно, что пластическая деформация протекает локализованным образом и характеризуется макроскопическими паттернами локализованного пластического течения, соответствующими автоволновым модам пластического течения [1-3,8,9]. Паттерны возникают на пределе текучести и далее закономерно эволюционируют в ходе деформации, приобретая форму, определяемую действующим на данной стадии процесса законом деформационного упрочнения.

Достоинством модели является ее способность объяснить рождение автоволнового макромасштаба локализованной пластичности $\lambda \approx 10^{-2} \mathrm{~m}$ за счет дислокационных явлений с их характерным микромасштабом порядка вектора Бюргерса $b \approx 10^{-10} \mathrm{~m}$, причем $b / \lambda \approx 10^{-8}$ [3]. Для объяснения используется механизм обменного (за счет упругих импульсов) взаимодействия между последовательно релаксирующими концентраторами напряжений, впервые предложенный в [10].

В рамках этой модели пластическая деформация рассматривается как эволюция паттернов локализованной пластичности в ходе процесса формоизменения [2,3]. Данная работа посвящена проблеме уточнения кинетики паттернов, реализующихся на разных стадиях деформации.

\section{2. Материалы, методика и экспериментальные данные}

2.1. Условия эксперимента. При выполнении экспериментальной части работы уточнялись особенности и детали развития паттернов локализованной пластичности в металлах. Для этого на испытательной 
машине „Instron-1185“ записывались деформационные кривые $\sigma(\varepsilon)(\sigma-$ напряжение, $\varepsilon-$ деформация $)$ выращенных методом Бриджмена монокристаллов чистых $\mathrm{Cu}, \mathrm{Ni}$ и высокомарганцовистой стали Гатфильда (Fe-12 wt.\% Mn-0.9 wt.\% C), а также поликристаллов хромоникелевой аустенитной стали $(0.1 \mathrm{wt} . \% \mathrm{C} ; 9.0 \mathrm{wt} . \% \mathrm{Ni}$; 22 wt.\% Cr). Размеры рабочих частей плоских образцов в этом случае составляли $45 \times 5 \times 2 \mathrm{~mm}$, а скорость движения подвижного захвата машины $V_{m}=10^{-5} \mathrm{~m} / \mathrm{s}$.

Паттерны локализованной пластичности регистрировались методом двухэкспозиционной спекл-фотографии [1], согласованным с записью кривых $\sigma(\varepsilon)$. Метод позволяет находить распределения компонент тензора пластической дисторсии

$$
\beta_{i j}=\nabla u(x, y)=\varepsilon_{i j}+\omega_{z}
$$

для разных моментов времени $t \sim \varepsilon$. В уравнении $(1)$ поле векторов смещения в плоском деформируемом образце, восстанавливаемое методом спекл-фотографии, $\varepsilon_{i j}$ - тензор деформации, $\omega_{z}-$ поворот вокруг оси $z$, нормальной к плоскости образца. При анализе экспериментальных данных использовались такие компоненты тензора (1) как локальные удлинение $\varepsilon_{x x}$, сдвиг $\varepsilon_{x y}$ и поворот $\omega_{z}[1]$. Кинетические характеристики автоволновых процессов пластического течения - длина автоволны и скорость ее распространения - определялись из графиков зависимостей координат максимумов локальных деформаций в образце от времени $X(t)$, называемых $X-t$-диаграммами.

Приведенные выше соображения о роли акустических импульсов в развитии локализованной пластичности заставили дополнить исследование деформации поликристаллических образцов аустенитной стали одновременной записью сигналов акустической эмиссии, сопровождающих деформацию образцов размером $100 \times 6 \times 2 \mathrm{~mm}$. Методика таких измерений, описанная в [11], позволяет определять координаты очагов генерации эмиссионных сигналов. Ее достоинства состоят в быстрой регистрации сигналов при деформации и в возможности регистрировать одновременно большое число сигналов, характеристики которых слабо зависят от изменения формы образцов при пластической деформации.

2.2. Экспе риментальные данные. Применение методики спекл-фотографии позволило наблюдать паттерны локализованной пластичности и регистрировать кинетику их развития. Как выяснилось, в исследованных материалах наблюдалась закономерная эволюция паттернов, форма которых определялась законом деформационного упрочнения [1]. В качестве наиболее интересных объектов в рамках настоящего исследоваия были выбраны и проанализированы устойчиво развивающиеся паттерны локализованной пластичности на стадиях [12]:

- площадки текучести (монокристаллы сплава $\mathrm{Fe}-\mathrm{Mn}-\mathrm{C})$,
- легкого скольжения (монокристаллы $\mathrm{Ni}$ ),

- линейного деформационного упрочнения (монокристаллы $\mathrm{Cu}$,

- параболического деформационного упрочнения (аустенитная сталь).

Этот выбор определен тем, что во всех случаях стадии деформационного упрочнения имели достаточно большую длину, что обеспечивало повышенную точность измерения их характеристик.

На площадке текучести, зарегистрированной на диаграмме деформации $\sigma(\varepsilon)$ монокристаллов сплава $\mathrm{Fe}-\mathrm{Mn}-\mathrm{C}$ с ориентацией оси растяжения [3777], паттерн локализованной пластичности представляет собой одиночный движущийся фронт локализованной деформации. Он соответствует зарождению и распространению по кристаллу полосы Людерса, сформированной из двойников деформации в первичной системе двойникования [211](111) [13]. В этом случае на протяжении площадки текучести вдоль образца перемещается одиночный фронт пластической деформации. Средняя скорость перемещения фронта, определяемая по наклону $X-t$-диаграммы, составила $\left\langle V_{a w}\right\rangle \approx 9 \cdot 10^{-6} \mathrm{~m} / \mathrm{s}$, (рис. 1, $a$, сплошная линия). Такой паттерн может рассматриваться как автоволна переключения [1,8,9], поскольку на фронте полосы Людерса среда переходит из упругого в пластически деформированное состояние.

Н а стадии легкого скольжения, наблюдавшейся при растяжении монокристаллов $\mathrm{Ni}$ с ориентацией оси растяжения [167], паттерн локализации пластического течения представляет собой два движущихся навстречу друг другу со средними скоростями $\left\langle V_{a w}^{+}\right\rangle \approx 3.1 \cdot 10^{-5} \mathrm{~m} / \mathrm{s}$ и $\left\langle V_{a w}^{-}\right\rangle \approx-2.2 \cdot 10^{-5} \mathrm{~m} / \mathrm{s}$ деформационных фронта. Момент их встречи отвечает окончанию стадии легкого скольжения. Наблюдаемая ситуация соответствует встречному движению двух автоволн переключения. Характер движения фронтов локализованной деформации в этом случае показан сплошными линиями на рис. $1, b$.

На стадии линейного деформационного упрочнения, наблюдавшейся в монокристаллах $\mathrm{Cu}$, паттерн локализованной деформации, представляет собой совокупность эквидистантных фронтов пластического течения, движущихся в одну сторону с постоянной скоростью $\left\langle V_{a w}\right\rangle \approx 8 \cdot 10^{-5} \mathrm{~m} / \mathrm{s}$. Это иллюстрируется на $X-t$-диаграмме, показанной на рис. 1, $c$. Наблюдаемый паттерн соответствует фазовой автоволне локализованного пластического течения $[1,8,9]$.

На стадии параболического деформационного упрочнения паттерн локализации пластического течения имеет вид стационарной системы фронтов пластического течения, т. е. в этом случае $\left\langle V_{a w}\right\rangle \approx 0$. Это показано сплошными линиями на рис. $1, d$, где приведены $X-t$-диаграммы для аустенитной стали на этой стадии деформации. Такой паттерн обычно рассматривается как стационарная диссипативная структура $[1,8,9]$.

Эти закономерности полностью соответствуют установленным ранее особенностям развития паттернов 

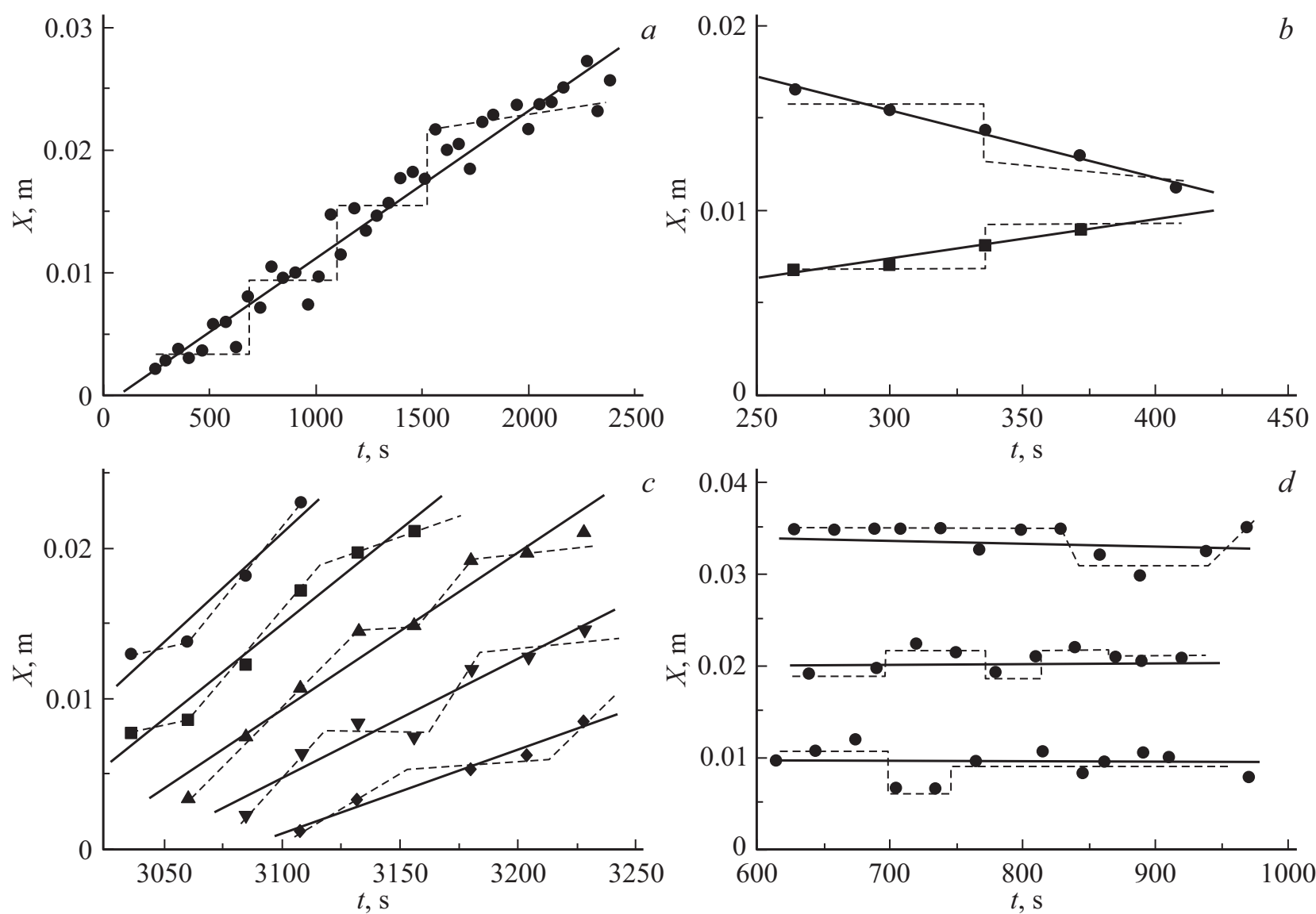

Рис. 1. $X-t$-диаграммы для паттернов локализованной пластичности: стадия площадки текучести $(a)$, стадия легкого скольжения $(b)$, стадия линейного деформационного упрочнения $(c)$, стадия параболического деформационного упрочнения $(d)$.

локализованной пластичности, характерным для всех исследованных материалов [14]. Можно записать общее для автоволн переключения, фазовых автоволн и стационарных диссипативных структур, соответствующих рис. $1, a-d$, уравнение $X-t$-диаграммы

$$
X_{n}=V_{a w} t+\lambda n,
$$

где $\lambda$ - длина автоволны, $n=0,1,2,3 \ldots$ номер наблюдаемого фронта. В этом виде оно применимо для фазовой автоволны (рис. 1,c). Для автоволны переключения $n=0$, и из (2) следует $X=V_{a w} t$ (рис. $\left.1, a\right)$, а для стационарной диссипативной структуры при $\left\langle V_{a w}\right\rangle \approx 0$ имеем $X_{n}=\lambda n$ (рис. $1, d$ ) для всех $t$. При записи уравнения (2) предполагается, что $V_{a w}=$ const для соответствующих стадий процесса деформации, т.е. оно описывает $X-t$-диаграммы, изображенные на рис. $1, a-d$ сплошными линиями.

\section{3. Обсуждение результатов}

Такой вариант описания пригоден только в случае усреднения кинематических характеристик автоволновых процессов пластического течения. Повышение точ- ности регистрации паттернов локализованной пластичности за счет выбора для анализа протяженных во времени стадий их развития позволило детализировать кинематику этих процессов.

3.1. Скоростной режим движения фронтов локализованной пластичности. Обратим внимание на возможность более точной аппроксимации $X-t$-диаграмм, показанных на рис. $1, a-d$, штриховыми ломаными линиями. В таком случае из рис. $1, a$ следует, что фронт полосы Людерса движется неравномерно: его кратковременные скачки чередуются с длительными остановками, т.е. в уравнении (2) следует полагать, что $0 \leq V_{a w} \leq V_{a w}^{\max }$. Таже закономерность отчетливо проявляется также при анализе данных рис. $1, b-d$, построенных для других стадий деформационного упрочнения.

Это указывает на необходимость изменить представление о характере скоростного режима процесса, обратив внимание на существенную неравномерность (скачкообразность) движения фронтов локализации пластического течения на всех стадиях процесса формоизменения. Неравномерность движения выражается в пространственно-временных колебаниях фронтов локализованного пластического течения на всех стадиях деформационного упрочнения. На это указывает ана- 

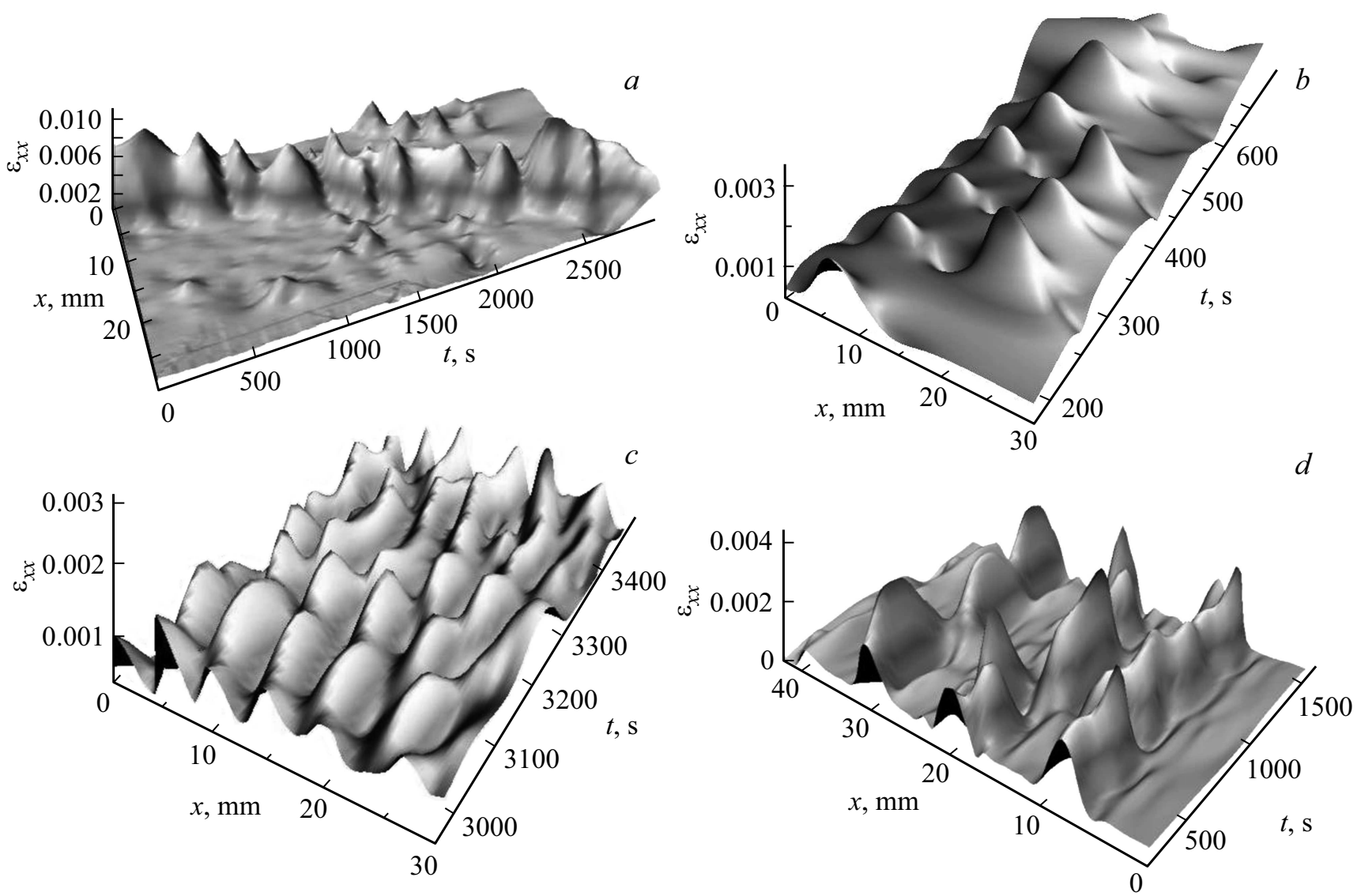

Рис. 2. Пространственно-временные распределения компоненты локальных удлинений для стадии площадки текучести (a), стадии легкого скольжения $(b)$, стадии линейного деформационного упрочнения $(c)$, стадии параболического деформационного упрочнения $(d)$.

лиз данных рис. 2, $a-d$, изображающих пространственно-временные распределения компоненты локального удлинения $\varepsilon_{x x}(x, t)$ на соответствующих стадиях деформационного упрочнения.

Особенно хорошо эта закономерность прослеживается при деформации Людерса, развивающейся в монокристалле сплава $\mathrm{Fe}-\mathrm{Mn}-\mathrm{C}$ (рис. 2,a). Видно, что величина $\varepsilon_{x x}$ колеблется по мере развития процесса во времени $t$ и вдоль оси образца $x$ относительно своего среднего значения. Временно́й период этих колебаний $T \approx 400 \mathrm{~s}$. При скорости движения фронта вдоль образца $\sim 9 \cdot 10^{-6} \mathrm{~m} / \mathrm{s}$, характерный пространственный масштаб $V_{a w} \cdot T \approx 3.5 \mathrm{~mm}$ соответствует многочисленным данным о ширине фронта полосы Людерса в разных материалах $[12,15,16]$.

Из данных рис. $2, b-d$ и $1, b-d$, следует, что аналогичные эффекты имеют место и для всех других стадий деформационного упрочнения. Иначе говоря, скачкообразность развития паттернов локализованной пластичности приобретает характер общей для процессов локализованного пластического течения закономерности.

Таким образом, новым результатом, полученным в настоящем исследовании и нуждающемся в объяснении, является неравномерность развития деформацион- ных процессов, связанных с движением фронтов локализованной пластичности на всех стадиях формоизменения.

3.2. Связь с распределениями компонент тензора де формаци и. Для понимания закономерностей скачкообразного развития фронтов локализованной пластичности рассмотрим более детально поведение других компонент тензора пластических деформаций на фронте полосы Людерса при росте общей деформации образца от 0.035 до 0.05. Как следует из рис. 3, вначале при общей деформации $\varepsilon_{t o t}=0.035$ максимумы распределений $\varepsilon_{x x}(x), \varepsilon_{x y}(x)$ и $\omega_{z}(x)$ совпадают (рис. $\left.3, a\right)$ при $x \approx 5 \mathrm{~mm}$. Затем при возрастании деформации до $\varepsilon_{t o t}=0.04$ максимумы $\varepsilon_{x y}(x)$ и $\omega_{z}(x)$ смещаются вправо на $\sim 2 \mathrm{~mm}$ по отношению к $\varepsilon_{x x}$ (рис. $\left.3, b\right)$. Наконец, при $\varepsilon_{t o t}=0.05$ компонента $\varepsilon_{x x}$ достигает максимума, в то время как распределения $\varepsilon_{x y}(x)$ и $\omega_{z}(x)$ расщепляются на две компоненты разного знака, смещенные относительно максимума распределения $\varepsilon_{x x}(x)$ на $\sim 3 \mathrm{~mm}$ влево и вправо (рис. 3,c).

Из сравнения рис. 1, $a$ и рис. 3 следует, что движение фронта Людерса начинается после перестройки распределений компонент $\varepsilon_{x x}(x), \varepsilon_{x y}(x)$ и $\omega_{z}(x)$ в зоне локализации в порядке, показанном на рис. 3. Совпаде- 

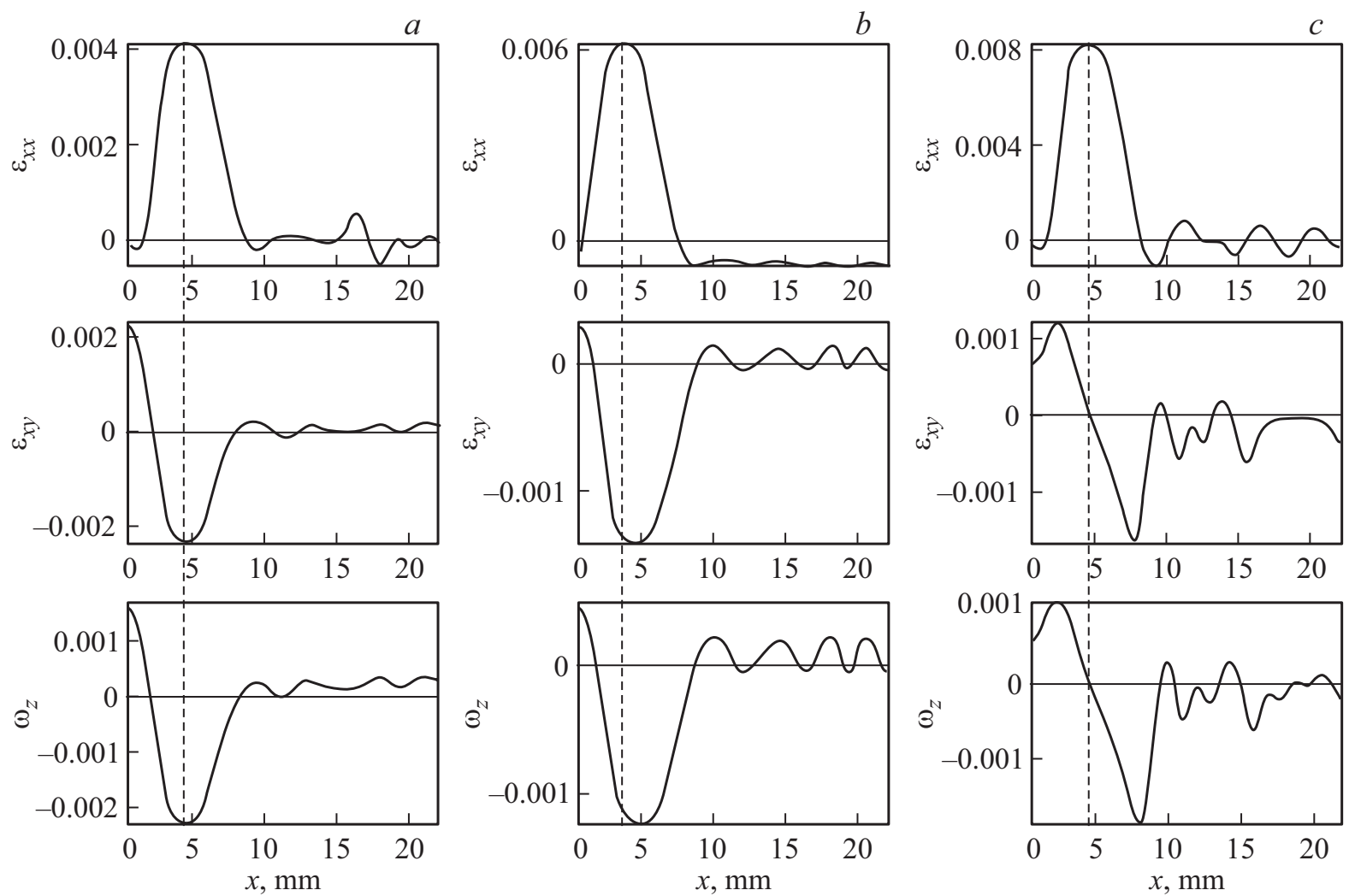

Рис. 3. Распределения компонент тензора пластической дисторсии на фронте полосы Людерса на площадке текучести при общей диформации $0.035(a), 0.04(b), 0.05(c)$.

ние экстремумов распределений всех компонент тензора пластической дисторсии (рис. 3,a) отвечает постоянству величины $X$. Скачкообразный прирост этой величины, отмеченный на рис. 1, $a$, напротив, соответствует пространственному разделению экстремумов распределений $\varepsilon_{x x}(x), \varepsilon_{x y}(x)$ и $\omega_{z}(x)$, показанному на рис. $3, c$. Как следует из данных рис. $3, c$, распределения $\varepsilon_{x y}(x)$ и $\omega_{z}(x)$ асимметричны: кроме разного знака деформаций, они отличаются амплитудой, причем фронт Людерса движется в сторону более высокого пика распределения, т. е. в сторону упруго деформированной части образца.

Можно полагать, что перераспределение компонент удлинения $\varepsilon_{x x}(x)$, сдвига $\varepsilon_{x y}(x)$ и поворота $\omega_{z}(x)$ инициирует скачкообразность развития пластического течения на всех стадиях пластического течения, который соответствует ломаным линиям на $X-t$-диаграммах, представленных на рис. $1, a-d$. Перестройки распределений компонент тензора дисторсии происходят несколько раз при движении очага деформации по образцу. Согласование максимумов локальных удлинений с максимумами сдвигов и поворотов в очаге локализации деформации и соответствующие перераспределению компонент тензора пластической дисторсии, описанному выше для площадки текучести, отмечено также на стадиях легкого скольжения, линейного и параболического деформационного упрочнения в исследованных материалах (рис. $1, b-d$ и $2, b-d$ ).
3.3. О роли импульсов акустической эмиссии. В соответствии с развиваемой моделью [2,3], импульсы акустической эмиссии формируют сигнальную систему, управляющую кинетикой процесса пластического течения и ответственную за явления самоорганизации деформируемой среды [1]. Возможность регистрации и лоцирования сигналов акустической эмиссии, сопровождающих разные стадии деформационного упрочнения, позволила подтвердить однозначное соответствие паттернов локализованного пластического течения и распределений источников акустической эмиссии [11].

В настоящей работе этот факт непосредственно следует из сравнения данных, представленных на рис. $2, d$ и рис. 4, $a$ и полученных при деформации поликристаллов аустенитной хромоникелевой стали на стадии параболического деформационного упрочнения. На этих рисунках представлены распределения компоненты $\varepsilon_{x x}(x, t)$ и плотности сигналов акустической эмиссии $J(x, t)$. Качественное сходство обсуждаемых распределений приводит к выводу о существовании между ними тесной взаимосвязи. Показанный на рис. 4 в аналог $X-t$-диаграммы, построенный для максимумов плотности распределения акустических сигналов $J(x, t)$, также подтверждает, что положения стационарных очагов локализованной пластичности и соответствующих им источников эмиссии на стадии параболического деформационного упрочнения совпадают. 

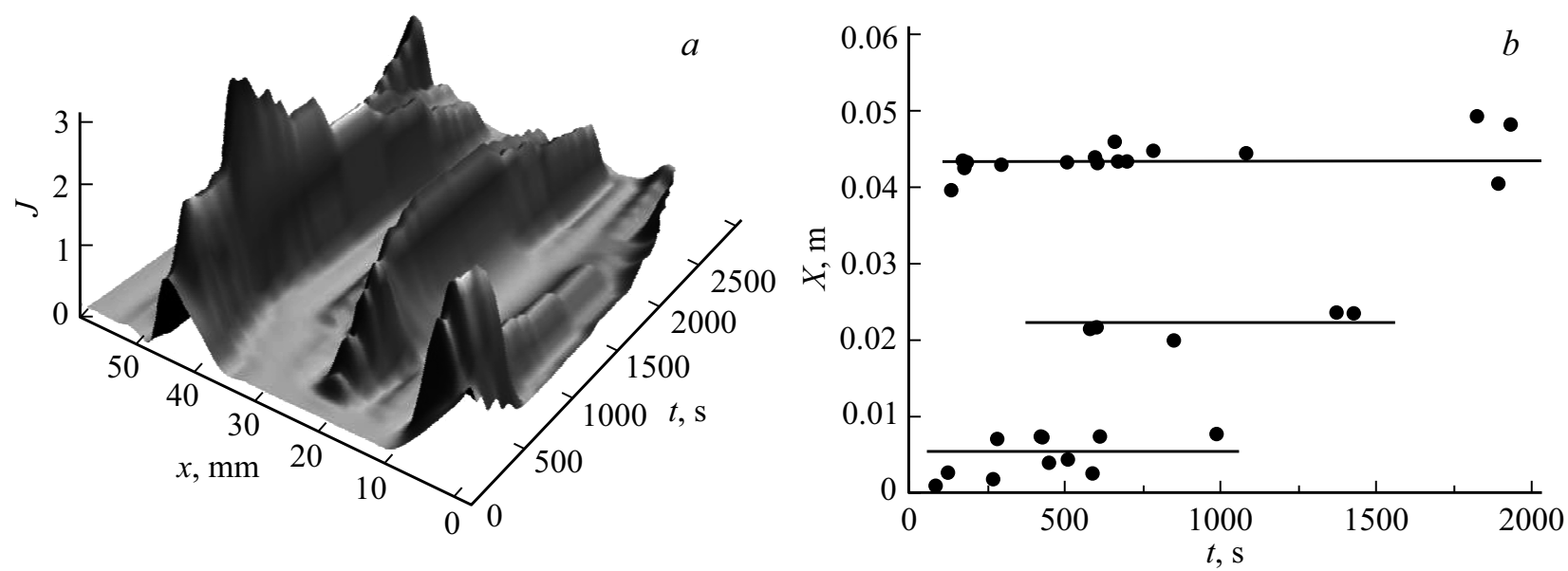

Рис. 4. Пространственно-временно́е распределение сигналов акустической эмиссии при параболическом деформационном упрочнении $(a)$ и координаты источников эмиссии $(b)$ для этого случая в поликристалле аустенитной стали.

Ранее эта закономерность была проанализирована в работе [11] на примере перехода деформационной кривой $\sigma(\varepsilon)$ от площадки текучести к стадии параболического деформационного упрочнения. При этом отмечена наследственность в образовании новых эмиссионных очагов, связанных с деформацией: они группируются в тех зонах образца, где ранее возникали максимумы акустической эмиссии при остановках фронтов Людерса.

Таким образом, данные измерений акустической эмиссии свидетельствуют о справедливости развиваемой модели процесса пластического течения. Наиболее важной особенностью развития паттернов локализованной деформации является их пространственно-временна́я периодичность, которая совпадает с такой же периодичностью генерации импульсов акустической эмиссии.

3.4. Механизм развития локализованных де формаций. Для понимания природы периодических процессов проанализируем данные рис. $2, a$, описывающие поведение величины $\varepsilon_{x x}(x, t)$ при деформации Людерса. Из них следует, что при развитии пластического течения начальный рост деформации сменяется ее спадом. Объясняя эту закономерность, положим, что деформация на фронте полосы Людерса есть термически активированный процесс [17], распространяющийся со скоростью, которая определяется нижним пределом текучести $\sigma_{y}^{(l)}$, как

$$
V_{a w}=V_{0} \exp \left(-\frac{U-\gamma \sigma_{y}^{(l)}}{k_{B} T}\right) .
$$

Здесь $V_{0}=$ const, $\gamma$ - активационный объем процесca [15], $U$ - высота потенциального барьера, $T$ температура, а $k_{B}$ - постоянная Больцмана. Таким образом, если в грубом приближении принять для площадки текучести условие $\sigma_{y}^{(l)}$, то из уравнения (3) следует, что при деформации Людерса фронт полосы движется co скоростью $V_{a w}=$ const как это показано на рис. $1, a$ сплошной линией.
Однако, как показывают результаты описанных выше экспериментов, деформация на площадке текучести развивается скачкообразно, что иллюстрируется штриховой линией на рис. $1, a$ и подтверждается известными данными о малых скачках напряжения на площадке текучести $[12,15,16]$. Причину развития такого режима можно понять из следующих соображений. Так как на площадке текучести деформационное упрочнение отсутствует, то можно считать, что напряжение течения близко к внутреннему напряжению $\sigma_{i n} \approx G b \rho^{1 / 2}$, взятому с обратным знаком. Тогда $\sigma_{y}^{(l)}=-\sigma_{i n}=-G b \rho^{1 / 2}$, где $G-$ модуль сдвига, а $\rho$ - плотность дислокаций на фронте Людерса [6], и из уравнения (3) следует

$$
\begin{aligned}
V_{a w} & =V_{0} \exp \left(-\frac{U-\gamma \sigma_{y}^{(l)}}{k_{B} T}\right) \\
& =V_{0} \exp \left(-\frac{U+\gamma G b \rho^{1 / 2}}{k_{B} T}\right) \sim \exp \left(-\rho^{1 / 2}\right) .
\end{aligned}
$$

Таким образом, скорость движения фронта Людерса при деформации на площадке текучести определяется поведением плотности дислокаций на фронте развивающейся полосы, т. е. формой зависимости $\rho(\varepsilon)$. Известно, что плотность дислокаций при деформации меняется экстремальным образом $[18,19]$, что, очевидно, качественно определяет аналогичный характер изменения скорости, согласно соотношению (4).

Косвенным образом в пользу справедливости такого предположения говорит сложный характер изменения компонент тензора пластической дисторсии, соответствующий началу развития фронта Людерса и показанный на рис. 3. Если полагать, что сдвиговая $\varepsilon_{x y}$ и поворотная $\omega_{z}$ компоненты этого тензора связаны с дислокационными механизмами деформации [6,19], то данные рис. 3 можно считать указанием на изменение плотности дислокаций вблизи фронта Людерса. 


\section{4. Заключение}

Таким образом, в настоящей работе путем сопоставления картин локализации пластической деформации и сигналов акустической эмиссии удалось продемонстрировать однозначное соответствие между различными модами автоволн локализации пластического течения, возникающими на разных стадиях деформационного упрочнения, и распределением источников акустической эмиссии, действующих в ходе этого же процесса. При этом процессы, протекающие на ранних стадиях деформирования, запоминаются деформированной средой и определяют развитие очагов локализации пластической деформации на последующих этапах нагружения.

Колебательный характер развития фронта Людерса при деформации на площадке текучести допускает возможность аналогии наблюдаемой неоднородности в структуре паттернов локализованной деформации и полос при развитии эффекта Портевена-Ле Шателье $[12,20,21]$.

\section{Список литературы}

[1] Л.Б. Зуев, В.И. Данилов, С.А. Баранникова. Физика макролокализации пластического течения. Наука, Новосибирск (2008). $327 \mathrm{c}$.

[2] L.B. Zuev. Phys. Wave Phenom. 20, 166 (2012).

[3] Л.Б. Зуев. ФММ 118, 810 (2017).

[4] A. Seeger, W. Frank. In: Non-linear Phenomena in Material Science. Eds. L.P. Kubin, G. Martin. Trans. Tech. Publ., N. Y. (1987). P. 125.

[5] Б.Б. Кадомцев. Динамика и информация. Редакция УФН, М. (1997). 397 c.

[6] Ж. Фридель. Дислокации. Мир, М. (1967). 643 с.

[7] Г.А. Малыгин. ФТТ 42, 69 (2000).

[8] V.I. Nekorkin, V.B. Kazantsev. Int. J. Bifurc. Chaos. 12, 2421 (2002).

[9] Е.П. Земсков, А.Ю. Лоскутов. ЖЭТФ 134, 406 (2008).

[10] Л.Б. Зуев, В.И. Данилов, В.В. Горбатенко. ЖТФ 65, 91 (1995).

[11] Е.С. Никитин, Б.С. Семухин, Л.Б. Зуев. Письма в ЖТФ 34, 70 (2008).

[12] J. Pelleg. Mechan. Properties Met. Springer, Dordrecht (2013). $634 \mathrm{p}$.

[13] I. Karaman, H. Sehitoglu, Y.I. Chumlyakov, H.J. Maier, I.V. Kireeva. Met. Mater. Trans. 32A, 695 (2001).

[14] Л.Б. Зуев. МФНТ 38, 1335 (2016).

[15] H.B. Sun, F. Yoshida, M. Ohmori, X. Ma. Mater. Lett. 57, 4535 (2003).

[16] Л.Б. Зуев. ПМТФ 58, 328 (2017).

[17] D. Caillard, J.L. Martin. Thermally Activated Mechanisms in Crystal Plasticity. Elsevier, Oxford (2003). 433 p.

[18] J.J. Gilman. J. Appl. Phys. 36, 2772 (1965).

[19] M. Zaiser, E.C. Aifantis. Int. J. Plasticity 22, 1432 (2006).

[20] A.A. Shibkov, M.F. Gasanov, M.A. Zheltov, A.T. Zolotov, V.I. Ivolgin. Int. J. Plasticity 86, 37 (2016).

[21] А.А. Шибков, М.А. Желтов, М.Ф. Гасанов, А.Е. Золотов. ФTT 60, 315 (2018).

Редактор Т.Н. Василевская 